\title{
Image Compression: An approach using Wavelet Transform and Modified FCM
}

\author{
G Boopathi \\ Assistant Professor of Computer Applications, \\ SNR Sons College (Autonomous), \\ Coimbatore 641006, India
}

\author{
Dr.S.Arockiasamy \\ Asst. Dean for Training \\ Head, Information Systems \\ University of Nizwa, \\ Sultanate of Oman
}

\begin{abstract}
In recent past, vector quantization has been observed as an efficient technique for image compression. In general, image compression reduces the number bits required to represent an image. The main significance of image compression is that the quality of the image is preserved. This in turn increases the storage space and thereby the volume of the data that can be stored. Image compression is the application of data compression technique on digital images. Wavelet Transform based image compression remain the most common among diverse techniques proposed earlier. Wavelet-based image compression provides considerable improvements in picture quality at higher compression ratios. A moment ago Artificial Neural Network has attained popularity in the field of image compression. This paper proposes a technique for image compression using modified Fuzzy C-Means (FCM) algorithm based vector quantization (VQ). The VQ codebook is generated by a modified FCM algorithm. The principal shortcoming of standard FCM algorithm is that the objective function does not think about the spatial dependence therefore it deal with image as the same as separate points. This proposed paper modifies the standard FCM algorithm that join together both the local spatial context and the non-local information into the standard FCM cluster algorithm using a novel dissimilarity index in place of the usual distance metric. Experiments are carried out in order to estimate the performance of the proposed modified FCM algorithm in image compression using standard image set. The results exposed the performance of our approach in perspective with other conventional image compression techniques

Keywords- Bits, Codebook, Neural Networks (NN), Modified Fuzzy C-Means (FCM) Algorithm, Vector Quantization (VQ), Image Compression, and Wavelet Transform
\end{abstract}

\section{INTRODUCTION}

Image compression is an indispensable characteristic of image processing without which it is difficult to transmit an image of large size through internet etc. Image compression is the relevance of data compression on digital images. In effect, the main purpose of image compression is to trim down redundancy of the image data in order to be able to store or transmit data in an efficient form. Image compression is reducing the size in bytes of a graphics file without debasing the quality of the image to an unacceptable level. The image compression approaches can be divided into two methods: lossless and lossy. The decrease in file size allows more images to be stored in a given amount of disk or memory space. It also reduces the time needed for images to be sent over the Internet or downloaded from Web pages. Therefore compression methods are being rapidly developed to compress large data files such as images, where data compression in multimedia applications has lately become very imperative [1].

In general wavelets are a mathematical tool for hierarchically decomposing functions. Numerous methods have been proposed earlier for lossy compression of digital images. Most common among them is Wavelet Transform based image compression. Image compression using Wavelet Transforms (WT) is a dominant method that is favoured by most of the researchers to get the compressed images at higher compression ratios with higher PSNR values [2]. Wavelet-based image compression provides significant improvements in picture quality at higher compression ratios. It is an accepted transform used for some of the image compression standards in lossy compression methods. Dissimilar to the discrete cosine transform, the wavelet transform is not Fourier-based and therefore wavelets do a superior job of handling discontinuities in data.

Artificial neural network (ANN) implementation in image processing applications has to some extent increased in recent years. Artificial Neural Networks have been applied to image compression problems, [3] due to their superiority over traditional methods when dealing with noisy or incomplete data. Artificial Neural networks seem to be well suited to image compression, as they have the ability to preprocess input patterns to produce simpler patterns with fewer components. Different types of Artificial Neural Networks have been trained to perform Image Compression. Feed-Forward Neural Networks, Self-Organizing Feature Maps, Learning Vector Quantizer Network, have been applied to Image Compression.

Numerous methods and techniques have been proposed previously for image compression using neural networks and wavelet transform. Image compression using wavelet transform and a neural network was suggested previously [4]. Artificial neural networks are popular in function approximation, due to their ability to approximate complicated nonlinear functions. Different image compression techniques were combined with neural network classifier for various applications [5] [6] [15]. Some recent papers show that the combination of neural network based approach and classical wavelet based approach leads to better compression ratio [7]. Combining the Wavelet Transform and Artificial Neural Networks utilizes the advantages of the two techniques thereby improving the 
compression ratio. They may also make sure the quality of the compressed image.

This paper proposes a technique for image compression using modified Fuzzy C-Means (FCM) algorithm based vector quantization. Fuzzy C-Means (FCM) Clustering algorithm is one of the popular approaches for assigning multi-subset membership values to pixels for either segmentation or other type of image processing. Vector Quantization (VQ) is useful in compressing data that arises in extensive range applications and it can realize better compression performance than any conventional coding techniques which based on the encoding of scalar quantities. The experimental results explore the performance of the proposed technique in image compression.

\section{RELATED WORK}

Image compression is an essential feature of image processing without which it is difficult to transmit an image of large size through internet etc. This section of the paper discusses some of the earlier work proposed on image compression using neural networks and wavelet transform.

Khashman et al. in [8] proposed a technique for compressing the digital image using neural networks and Haar Wavelet transform. The aim of the work presented within the paper was to develop an optimum image compression system using haar wavelet transform and a neural network. With Wavelet transform based compression, the quality of compressed images is typically high, and the option of a perfect compression ratio is complicated to formulate as it varies depending on the content of the image. They proposed that neural networks can be trained to ascertain the non-linear relationship between the image intensity and its compression ratios in search for an optimum ratio. Moreover their paper suggested that a neural network could be trained to be familiar with an optimum ratio for Haar wavelet compression of an image upon presenting the image to the network. The method utilized Haar compression with nine compression ratios and a supervised neural network that learns to correlate the grey image intensity (pixel values) with a single optimum compression ratio. Two neural networks receiving different input image sizes are developed in their work and a comparison between their performances in finding optimum Haar-based compression was presented.

A method of still image compression was put forth by Wilford Gillespie in [9]. The fundamental approach to image compression consists of a number of key steps. They are wavelet packet decomposition, quantization, organization of vectors, neural networks approximation or storage, and lossless encoding and reduction. As an initial stage of image compression, the image is put through several layers of wavelet packet decomposition. The results of the decomposition are then divide or processed in some way, depending on the method. Integer quantization is performed on all of the decomposed wavelet sections. The quantization value is the determining factor of quality. A quantization value of 1 is near lossless quality, although little to no compression is achieved. This is accomplished by taking each section and dividing it by a set value and rounding to the nearest integer. There are many ways to systematize a tree of decomposition sections. Three methods were tried with this compression scheme. The type of neural network used in their approach was a two-layer feed-forward network with a standard back propagation learning function. At last, the entire data stream is taken and is processed by a run length encoded (RLE) method and saved in a lossless state using the ZIP file format.

Dandawate et al. in [10] described an approach for the design for vector Quantizer using neural networks and wavelet for image compression. Their approach was based on Kohonen's selforganizing feature maps (SOFM) and wavelet transform. They generated the code vectors by evaluating the characteristics of the particular image sub samples, which are determined through accurate mathematical operations and training the selected samples by Kohonen's SOFM artificial neural network with adjustable learning rate and initializations conditions followed by application of discrete wavelet transform (DWT). The testing of the generated codebook was done with variety of images and the compression performance was evaluated by using objective and subjective quality measures. They also suggested that codebooks can also be designed using various mother wavelet and performance can be evaluated. Run length coding (RLE) coding technique can be applied in order to reduce the file size further. Their proposed design is such that it can be implemented with less complexity and cost using VLSI techniques.

A Novel Hybrid Image Compression Technique was projected by Dwivedi et al. in [11]. The technique inherits the properties of localizing the global spatial and frequency correlation from wavelets and classification and functional approximation tasks from modified forward-only counter propagation neural network (MFOCPN) for image compression. In other words they have integrated wavelet transform based image compression to MFOCPN based image compression scheme. As a substitute of passing whole pixel values of Image one can pass the important wavelet coefficients obtained after applying wavelet transform to Image. Thus, this combination provides a better compression because at one stage compression is achieved by wavelet transform and in next stage the compression can be done with MFO-CPN. The MFO-CPN was used for predicting wavelet coefficients, and training is done for each wavelet level and subband which is obtained after applying the threshold. Several benchmark test images are used to investigate usefulness of the proposed technique. Experimental results of the proposed technique showed an enhancement in performance measures with respect to decoded picture quality and compression ratios, compared to the existing wavelet and neural network based image compression techniques.

Amar et al. in [12] proposed a wavelet networks approach for image compression. Wavelet networks are a combination of radial basis function (RBF) networks and wavelet decomposition, where radial basis functions were replaced by wavelets. The wavelet network is a combination of wavelets and neural networks. The network can be considered composed of three layers: a layer with $\mathrm{N}_{\mathrm{i}}$ inputs, a hidden layer with $\mathrm{N}_{\mathrm{w}}$ wavelets and an output linear neuron receiving the weighted outputs of wavelets. Both input and output layers are fully connected to the hidden layer. Moreover they used a feed forward propagation algorithm from input neurons to output neurons. The main similarity between the proposed wavelet network and the neural network is that both networks calculate a linear combination of nonlinear functions to adjust parameters. These nonlinear functions depend on adjustable parameters (dilations and translations). During training stage the weights, 
dilations and translations parameters, are iteratively adjusted to minimize the network error. They used a quadratic cost function to evaluate this error. In order to test the robustness of their approach, they have implemented and compared the results with some other approaches based on neural networks (MLP).

A Neuro-Wavelet based approach for image compression was put forth by Singh et al. in [13]. Images have large data quantity. For storage and transmission of images, high efficiency image compression methods are under wide attention. They proposed a neuro- wavelet based model for image compression which combines the advantage of wavelet transform and neural network. Images are decomposed using wavelet filters into a set of sub bands with different resolution corresponding to different frequency bands. Different quantization and coding schemes are used for different sub bands based on their statistical properties. The coefficients in low frequency band are compressed by differential pulse code modulation (DPCM) and the coefficients in higher frequency bands are compressed using neural network. Using their proposed scheme one can accomplish satisfactory reconstructed images with large compression ratios. Their experimental results revealed that their proposed technique of image compression out performed some of the conventional image compression approaches.

Wang et al. in [14] described a novel method of encoding an image without blocky effects. The proposed method incorporates the wavelet transform and a self-development neural network-the Vitality Conservation (VC) network to realize considerable improvement in image compression performance. The implementation consists of three steps. The image is initially decomposed at different scales using wavelet transform to achieve an orthogonal wavelet representation of the image. Each band can be consequently processed in parallel. In the second step, the discrete Karhunen-Loeve transform is used to extract the principal components of the wavelet coefficients. Thus, the processing speed can be much faster than otherwise. Finally, results of the second step are used as input to the VC network for vector quantization. Their simulation results showed that such an implementation can, in much less time, achieve superior reconstructed images to other methods.

\section{PROPOSED APPROACH}

\subsection{Wavelets for image compression}

Wavelet transform make use of both the spatial and frequency correlation of data by dilations (or contractions) and translations of mother wavelet on the input data. It supports the multiresolution analysis of data i.e. it can be applied to various scales according to the details required, which allows progressive transmission and zooming of the image without the need of extra storage. One more encouraging feature of wavelet transform is its symmetric behavior that is both the forward and the inverse transform has the same complexity, building fast compression and decompression routines. Its characteristics well suited for image compression comprise the ability to take into account of Human Visual System's (HVS) characteristics, very good energy compaction capabilities, robustness under transmission, high compression ratio etc.

The implementation of wavelet compression method is very similar to that of subband coding scheme: the signal is decomposed using filter banks. The output of the filter banks is down-sampled, quantized, and encoded. The decoder decodes the coded representation, up-samples and recomposes the signal.
Wavelet transform divides the information of an image into approximation and detail subsignals. The approximation subsignal illustrates the general trend of pixel values and other three detail subsignals show the vertical, horizontal and diagonal details or changes in the images. If these details are very small (threshold) then they can be set to zero without significantly changing the image. The greater the number of zeros the greater the compression ratio. If the energy retained (amount of information retained by an image after compression and decompression) is $100 \%$ then the compression is lossless as the image can be reconstructed exactly. This occurs when the threshold value is set to zero, meaning that the details have not been changed. If any value is changed then energy will be lost and thus lossy compression occurs. As more zeros are obtained, more energy is lost. Therefore, a balance between the two needs to be found out.

\subsubsection{Fuzzy C-Means Algorithm}

Fuzzy C-Means (FCM) Clustering algorithm is one of the popular approaches for assigning multi-subset membership values to pixels for either segmentation or other type of image processing [16]. In general, FCM algorithm proceeds by iterating the two necessary conditions until a solution is reached. Each data point will be coupled with a membership value for each class after FCM clustering. The aim of FCM is to calculate the cluster centers and to generate the class membership matrix. In other words, it assigns a class membership to a data point, depending on the similarity of the data point to a particular class relative to all other classes. The class membership matrix is a $\mathrm{cXN}$ matrix; in which $\mathrm{c}$ is the number of groups and $\mathrm{N}$ is the number of samples. Let $X=\left\{x_{1} \ldots \ldots x_{n}\right\}$ be the training set and $c \geq 2$ be an integer. A fuzzy c-partition of ' $\mathrm{X}$ ' can be represented by a matrix, $\mathrm{U}=\left\{\mu_{\mathrm{ik}}\right\} \in \mathrm{R}^{\mathrm{cXN}}$. U can be used to describe the cluster structure of $X$, by evaluating $\mu_{\mathrm{ik}}$, as a degree of membership of $\mathrm{x}_{\mathrm{k}}$ to cluster $\mathrm{i}$. The codebook vectors are evaluated by minimizing the distortion measure given by the following equation,

$$
\text { Minimize: } \mathrm{J}_{\mathrm{m}}(\mathrm{U}, \mathrm{v})=\sum_{k=1}^{N} \sum_{i=1}^{c}\left(\mu_{k i}\right)^{m}\left\|X_{k}-v_{i}\right\|^{2} A
$$

Where $X=\left\{x_{1}, x_{2}, \ldots x_{N}\right\} \subset R^{N}$ in a dataset, $c$ is the number of clusters in $\mathrm{X}: 2 \leq \mathrm{c}<\mathrm{N}, \mathrm{m}$ is a weighting exponent: $1 \leq \mathrm{m}<\infty$, $\mathrm{U}=\left\{\mu_{\mathrm{ik}}\right\}$ is the fuzzy c-partition of $\mathrm{X},\left\|X_{k}-v_{i}\right\| A$ is an induced a-norm of $\mathrm{R}^{\mathrm{N}}$, and $\mathrm{A}$ is a positive-definite $(\mathrm{NXN})$ weight matrix.

A conventional FCM algorithm includes the following steps,

1. Initially values are set for the parameters like c, A, $\mathrm{m}, \varepsilon$, and the loop counter' $\mathrm{t}$ ' is set to 1 ,

2. As a next step it is necessary to create a random cXN membership matrix $\mathrm{U}$,

3. The cluster centers are then evaluated using the following equation,

$$
v_{i}^{(t)}=\sum_{k=1}^{N}\left(\mu_{k i}^{(t)}\right)^{m} X_{k} / \sum_{k=1}^{N}\left(\mu_{k i}^{(t)}\right)^{m}
$$

4. The membership matrix is updated periodically with the help of the following equation, 


$$
\mu_{k i}^{(t+1)}=\left[\sum_{j=1}^{c}\left(\frac{d_{k i}}{d_{k j}}\right)^{\frac{2}{m-1}}\right]^{-1}
$$

Where $d_{k i}$ is given by $\left\|X_{k}-v_{i}^{(t)}\right\| A$

5. If $\max \left\{\left|\mu_{k i}^{(t)}-\mu_{k i}^{(t-1)}\right|\right\}_{>\varepsilon, \text { increment' } \mathrm{t}^{\prime} \text { and go to }}$ step 3 .

\subsubsection{Modified Fuzzy C-Means Clustering Technique for image compression}

The main drawback of standard FCM algorithm is that the objective function does not consider the spatial dependence therefore it deal with image as the same as separate points. In order to decrease the noise effect during image compression, the proposed method incorporates both the local spatial context and the non-local information into the standard FCM cluster algorithm using a novel dissimilarity index in place of the usual distance metric. Therefore a modified FCM algorithm is used to compress the image in our proposed paper. The non-local means algorithm [17] [18] tries to take advantage of the high degree of redundancy in an image. The membership value decides the compression results and hence the membership value is evaluated by the distance measurement denoted as $d_{k i}$. Therefore the approach modifies the distance measurement parameter which is readily influenced by local and non-local information.

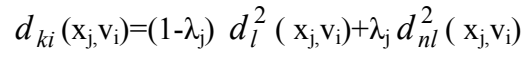

where $d_{1}$ stands for the distance measurement influenced by local information, and $\mathrm{d}_{\mathrm{nl}}$ stands for the distance measurement influenced by non-local information, $\lambda_{j}$ with the range from zero to one, is the weighting factor controlling the tradeoff between them.

The distance measurement influenced by the local measurement $d_{1}$ is given by,

$$
d_{1}^{2}\left(x_{j}, v_{i}\right)=\frac{\sum_{x_{k} \in N_{j}} \omega_{1}\left(x_{k}, x_{j}\right) d^{2}\left(x_{k}, v_{i}\right)}{\sum_{x_{k} \in N_{i}} \omega_{1}\left(x_{k}, x_{j}\right)}
$$

Where $d^{2}\left(\mathrm{x}_{\mathrm{j},} \mathrm{v}_{\mathrm{i}}\right)$ is the Euclidean distance measurement, $\omega \mathrm{l}\left(\mathrm{x}_{\mathrm{k}}\right.$,

$\mathrm{x}_{\mathrm{j}}$ ) is the weight of each pixel in $\mathrm{N}_{\mathrm{i}}$.

The distance measurement influenced by non-local information $\mathrm{d}_{\mathrm{nl}}$ is computed as a weighted average of all the pixels in the given image I,

$$
d_{\mathrm{nl}}^{2}\left(x_{j}, v_{i}\right)=\sum_{x_{k} \in I} \omega_{\mathrm{nl}}\left(x_{k}, x_{j}\right) d^{2}\left(x_{k}, v_{i}\right)
$$

Modified FCM algorithm goes through the following steps,

1. Set the number of clusters ' $c$ ' and the index of fuzziness ' $m$.' Also initialize the fuzzy cluster Centroid vector ' $v$ ' randomly and set $\varepsilon>0$ to a small value,

2. Set the neighborhood size and the window size includes the evaluation of cluster centers and membership matrix,
3. Evaluate the modified distance measurement using the equation mentioned as $d_{k i}\left(\mathrm{x}_{\mathrm{j}}, \mathrm{v}_{\mathrm{i}}\right)$,

4. Update the membership matrix and the distance measurement.

\subsubsection{FCM based vector quantization}

The reference vector or the average vector from each cluster is calculated using the FCM algorithm. The vector quantization codebook can be designed with the assistance of the outcome of FCM process. The outcome of the FCM process is a membership matrix and a set of reference vectors. The training vector will be encoded with this codebook.

\section{EXPERIMENTAL RESULTS}

In order to evaluate the performance of our proposed approach of image compression using modified FCM algorithm based vector quantization two standard images are considered. The work is implemented using MATLAB. Lena and Cameraman are the two standard images used to explore the performance of the proposed approach of image compression. The experiments are carried out with the number of clusters of 4,16 and 64 . For generating different codebooks, two images namely Lena and Cameraman images were used. In the testing phase the images used are in Figure 1, some reconstructed and original images are shown. Different PSNR and compression ratios as obtained from the testing phase are listed in table-1. All of the experimental images were of $512 \times 512$ pixels.

The evaluation of the proposed approach in image compression was performed using the following measures,

$$
\begin{gathered}
P S N R=10 \log _{10}\left[\frac{255^{2}}{M S E}\right](d B) \\
M S E=\frac{1}{3 M X N} \sum_{i=0}^{M-1} \sum_{j=0}^{N-1} \sum_{k=1}^{3}\left\{X_{k}(j, i)-\bar{X}_{k}(j, i)\right\}^{2}
\end{gathered}
$$

The experimental results that evaluate the performance of the proposed approach by comparing it with the standard FCM algorithm are tabulated. Table 1 shows the experimental results applied for three band (24 bits) Lena image and table 2 shows the experimental results of modified FCM applied for Cameraman image

Table.1 Experimental Results for Lena Image

\begin{tabular}{|c|c|c|c|c|}
\hline \multirow{2}{*}{$\begin{array}{l}\text { Number } \\
\text { of } \\
\text { Clusters }\end{array}$} & \multicolumn{2}{|c|}{ PSNR $(\mathrm{dB})$} & \multicolumn{2}{c|}{ MSE } \\
\cline { 2 - 5 } & $\begin{array}{c}\text { Modified } \\
\text { FCM }\end{array}$ & $\begin{array}{c}\text { Standard } \\
\text { FCM }\end{array}$ & $\begin{array}{c}\text { Modified } \\
\text { FCM }\end{array}$ & $\begin{array}{c}\text { Standard } \\
\text { FCM }\end{array}$ \\
\hline 4 & 22.650 & 20.125 & 405.180 & 407.520 \\
\hline 8 & 25.654 & 23.987 & 345.547 & 357.657 \\
\hline 16 & 27.410 & 24.540 & 118.560 & 121.325 \\
\hline 32 & 30.087 & 26.974 & 91.111 & 82.364 \\
\hline 64 & 32.250 & 29.100 & 49.750 & 53.650 \\
\hline
\end{tabular}

Figure 1 
(a) Sample Lena Image (b) Resultant image by existing approach (c) Resultant image by proposed approach

(a)

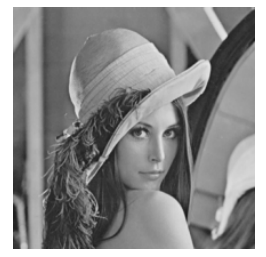

(b)
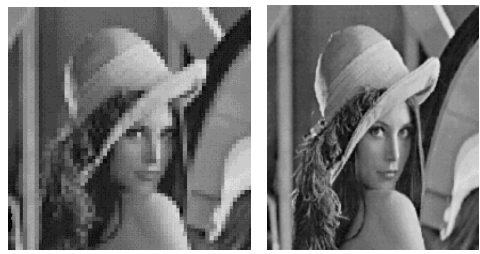

Table. 2 Experimental Results for Cameraman Image

\begin{tabular}{|c|c|c|c|c|}
\hline \multirow{2}{*}{$\begin{array}{c}\text { Number of } \\
\text { Clusters }\end{array}$} & \multicolumn{2}{|c|}{ PSNR (dB) } & \multicolumn{2}{c|}{ MSE } \\
\cline { 2 - 5 } & $\begin{array}{c}\text { Modified } \\
\text { FCM }\end{array}$ & $\begin{array}{c}\text { Standard } \\
\text { FCM }\end{array}$ & $\begin{array}{c}\text { Modified } \\
\text { FCM }\end{array}$ & $\begin{array}{c}\text { Standard } \\
\text { FCM }\end{array}$ \\
\hline 4 & 23.500 & 21.365 & 406.100 & 408.500 \\
\hline 8 & 25.167 & 24.982 & 247.685 & 257.652 \\
\hline 16 & 28.680 & 25.450 & 117.840 & 120.250 \\
\hline 32 & 39.225 & 26.957 & 85.364 & 92.365 \\
\hline 64 & 31.230 & 28.500 & 48.525 & 51.400 \\
\hline
\end{tabular}

(a)

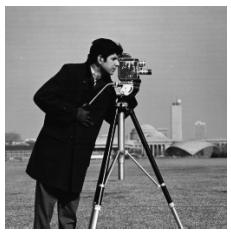

(b)

Figure 2

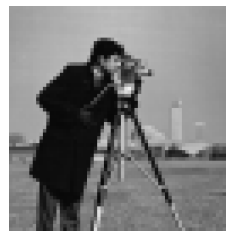

(c)

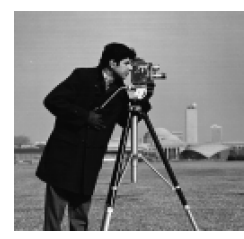

(a) Cameraman Image (b) Resultant image by existing approach (c) Resultant image by proposed approach

The experimental results of the proposed approach of image compression using modified Fuzzy C-Means algorithm based vector quantization codebook generation revealed the fact that the compression ratio of the proposed approach is high when comparing with other conventional image compression techniques. The Lena and Cameraman Image given for compression and the decompressed image by the existing and the modified approaches are presented below.

Figure 1 and 2 shows the sample input image of Lena and Cameraman respectively and the retrieved image when the existing and proposed approaches used for compression.

The experimental results of the proposed approach of image compression using modified Fuzzy C-Means algorithm based vector quantization codebook generation revealed the fact that the compression ratio of the proposed approach is high when comparing with other conventional image compression techniques. The Lena and Cameraman Image given for compression and the decompressed image by the existing and the modified approaches are presented below.

\section{CONCLUSION}

FCM is an extensively accepted clustering method and has been widely applied for image compression. This proposed paper introduced a modified Fuzzy C-Means algorithm for image compression. This variation overcomes the restraint of the standard FCM algorithm. The Vector Quantization (VQ) codebook is generated by a modified FCM algorithm. This proposed paper modifies the standard FCM algorithm that integrates both the local spatial context and the non-local information into the standard FCM cluster algorithm using a novel dissimilarity index in place of the usual distance metric. The membership value decides the compression results and hence the membership value is evaluated by the distance measurement. In order to estimate the performance of modified FCM based vector quantization for image compression some standard image set are considered. The experimental results revealed the fact that the compression ratio of the proposed approach is high when comparing with other conventional image compression techniques. The major limitation of the proposed approach is that it is computationally expensive, and this may limit its applicability to large $3 \mathrm{D}$ volume data. Implementation of some suppression technique during the process of iteration helps to overcome this limitation. The future work relies on implementing a suppression technique that can reduce the number of iterations and increase convergence speed of our proposed algorithm effectively.

\section{REFERENCES}

[1] M. J. Nadenau, J. Reichel, and M. Kunt, "Wavelet Based Color Image Compression: Exploiting the Contrast Sensitivity Function," IEEE Transactions Image Processing, Vol. 12, no.1, Pp. 58-70, 2003.

[2] K. H. Talukder, and K. Harada, "Haar Wavelet Based Approach for Image Compression and Quality Assessment of Compressed Image," IAENG International Journal of Applied Mathematics, 2007.

[3] M. Egmont-Petersen, D. de. Ridder, and Handels, "Image Processing with Neural Networks - a review," Elsevier, Journal on Pattern Recognition, Vol. 35, no. 10, Pp. 22792301, 2002.

[4] S. Osowski, R. Waszczuk, and P. Bojarczak, "Image compression using feed forward neural networksHierarchical approach," Lecture Notes in Computer Science, Book Chapter, Springer-Verlag, Vol. 3497, Pp. 1009-1015, 2006.

[5] M. Liying, and K. Khashayar, "Adaptive Constructive Neural Networks Using Hermite Polynomials for Image Compression," Lecture Notes in Computer Science, Springer-Verlag, Vol. 3497, Pp. 713-722, 2005.

[6] B. Karlik, "Medical Image Compression by Using Vector Quantization Neural Network," ACAD Sciences press in Computer Science, Vol. 16, no. 4, Pp. 341-348, 2006.

[7] Christopher J. C. Burges, Henrique S. Malvar, and Patrice Y. Simard, "Improving Wavelet Image Compression with Neural Networks," MSR-TR-2001-47, Pp. 1-18, August 2001.

[8] Adnan Khashman, and Kamil Dimililer, "Image 
Compression using Neural Networks and Haar Wavelet," WSEAS Transactions on Image Processing, Vol. 4, no. 5, Pp. 330-339, 2008.

[9] Wilford Gillespie, "Still Image Compression using Neural Networks," 2005.

[10] Y. H. Dandawate, T. R. Jadhav, A. V. Chitre, and M. A. Joshi, "Neuro-Wavelet based vector quantizer design for image compression," Indian Journal of Science and Technology, Vol. 2, no. 10, Pp. 56-61, 2009.

[11] Ashutosh Dwivedi, N. Subhash Chandra Bose, Ashiwani Kumar, Prabhanjan Kandula, Deepak Mishra, and Prem K Kalra, "A Novel Hybrid Image Compression Technique: Wavelet-MFOCPN," In Proceedings of ASID'06, Pp. 492495, 2006.

[12] C. Ben Amar, and O. Jemai, "Wavelet Networks Approach for Image Compression," ICGST, 2003.

[13] V. Singh, N. Rajpal, and K. S. Murthy, "Neuro-Wavelet Based Approach for Image Compression," Computer Graphics, Imaging and Visualization, CGIV apos'07, Pp. 280-286, 2007.

[14] Jung-Hua Wang, and Ker-Jiang Gou, "Image compression using wavelet transform and self-development neural network," IEEE International Conference on Systems, Man, and Cybernetics, Vol. 4, Pp. 4104-4108, 1998.

[15] Kussay Nugamesh Mutter, Zubir Mat Jafri, and Azlan Bin Abdul Aziz, "Hybrid Hopfield Neural Network, Discrete Wavelet Transform and Huffman Coding for Image Recognition," IJCSNS International Journal of Computer Science and Network Security, Vol. 9, no. 6, Pp. 73-78, 2009.
[16] J. C. Bezdek, "Pattern Recognition with Fuzzy Objective Function algorithms," Plenum Press, New York, 1981.

[17] A. Buades, B. Coll, and J. -M. Morel, "A non-local algorithm for image denoising," In CVPR, Vol. 2, Pp. 60$65,2005$.

[18] A. Buades, B. Coll, J. -M. Morel, "On image denoising methods,” Technical Report 2004-15, CMLA, 2004.

Mr.G.Boopathi received the Master of Computer Application in 1998. He is currently working towards the Ph.D. Degree in the Department of Computer Application, Karunya University, Coimbatore. His research interest includes Image Compression using Neural Networks. He is life member of CSI.

Dr.S.Arockiasamy, Head, Information System, University of Nizwa. He received M.Sc., M.Phil. and Ph.D. in Computer Science. He is specialized in Applications of Image processing. He has published a considerable number of Research papers and articles in various leading International journals and International Conferences. He is also Chief Editor of a Quarterly Computer Magazine CSI TIMES (Computer Society of India) and Voice of IT. 